Résumés des conférences et travaux

146 | 2015

2013-2014

\title{
Histoire et sociétés du Vietnam classique
}

\section{Histoire et sociétés du Vietnam classique}

Conférences de l'année 2013-2014

\section{Philippe Papin}

\section{OpenEdition \\ Journals}

Édition électronique

URL : https://journals.openedition.org/ashp/1751

DOI : 10.4000/ashp.1751

ISSN : 1969-6310

Éditeur

Publications de l'École Pratique des Hautes Études

Édition imprimée

Date de publication : 1 septembre 2015

Pagination : 293-296

ISSN : 0766-0677

Référence électronique

Philippe Papin, « Histoire et sociétés du Vietnam classique », Annuaire de l'École pratique des hautes études (EPHE), Section des sciences historiques et philologiques [En ligne], $146 \mid 2015$, mis en ligne le 06 octobre 2015, consulté le 07 juillet 2021. URL : http://journals.openedition.org/ashp/1751 ; DOI : https://doi.org/10.4000/ashp.1751 


\title{
HISTOIRE ET SOCIÉTÉS DU VIETNAM CLASSIQUE
}

\author{
Directeur d'études : M. Philippe PAPIN
}

Programme de l'année 2013-2014 : I. Éléments épigraphiques pour une histoire économique et financière des campagnes aux XVII et XVIII ${ }^{e}$ siècles. - II. Études des donations pieuses à l'époque moderne (suite).

Les conférences de l'année 2013 et du tout début 2014 ont permis d'achever le cycle consacré à la mesure comptable des donations bouddhistes. Après l'étude des dons en numéraire, laquelle inclut le calcul de la dépréciation de la monnaie, on a abouti au tracé d'une courbe générale indiquant, sur la base des textes examinés lors du séminaire, l'évolution de la valeur de l'argent offert à la pagode ou au conseil communal - ou aux deux à la fois $-\mathrm{du} \mathrm{XVII}^{\mathrm{e}}$ au XIX ${ }^{\mathrm{e}}$ siècles. Il a ensuite été question des terres offertes par les donateurs. En cette matière, le calcul est plus simple puisque la valeur d'usage est prépondérante et les rendements agricoles à peu près stables sur la période, ou du moins très peu différents. Néanmoins, le même travail d'estimation de la valeur des dons a été mené, et il a débouché lui aussi sur une courbe indicatrice de l'évolution générale.

Chemin faisant, un thème intéressant est apparu : non plus la valeur vénale mais le nombre et la taille des parcelles données. De fait, en règle générale, le don foncier consistait en plusieurs parcelles ajoutées les unes aux autres. Le total moyen s'élevait au début du $\mathrm{XIX}^{\mathrm{e}}$ siècle à une douzaine de sào par contrat de donation, soit moins d'un demi-hectare, mais divisé en trois, quatre, cinq petits morceaux, allant jusqu'à plus de dix dans certains cas. Concrètement, ces étroits lopins étaient des bouts de champs ou de jardins, des terres situées trop loin pour que l'exploitation fût rentable, des reliquats après découpe de propriété, des saillants de parcelles, des terrains isolés ou pentus. Autrement dit, on ne donnait pas toujours le meilleur, et même on donnait ce dont la privation dérangeait peu. Et pourtant, ce sont ces aliénations de parcelles microscopiques qui ont permis de restructurer en permanence le finage villageois. La pagode ou les autorités villageoises, en réunissant les bouts, en les échangeant contre d'autres, et en achetant ce qui manquait le cas échéant, ont pu reconstituer des rizières et des champs exploitables, c'est-à-dire rationaliser et remembrer la propriété agricole.

D'autre part, la terre offerte devenait $k y$ diền 忌田, «terre de commémoration funèbre ", ce qui implique qu'elle acquérait un statut public : elle tombait dans l'escarcelle de la propriété collective. Alors que tout le mouvement historique depuis le $\mathrm{XV}^{\mathrm{e}}$ siècle va vers la privatisation des finages - deux tiers des terres vietnamiennes sont de statut privé au début du XIX ${ }^{\mathrm{e}}$ siècle, et $90 \%$ dans les villages rizicoles du delta - la donation pieuse a permis au clergé et aux autorités civile de reconstituer leur patrimoine collectif, ou du moins d'en limiter l'érosion. Et comme c'est cette propriété collective qui était divisée en lots de terre distribués gratuitement aux pauvres du villages, 
pour une durée allant de trois à six ans, la donation a bel et bien participé, par dérivation d'usage, à la consolidation des amortisseurs sociaux ayant fait la force des campagnes durant toute l'époque moderne.

La suite des conférences a traité des aspects financiers de la donation en les replaçant dans un contexte économique plus large. Dans tous les cas et à toutes les époques, il est évident que le contenu financier de la donation a joué un rôle primordial. On peut même facilement supposer - et parfois prouver - qu'une part du don allait directement dans la caisse communale ou celle de la pagode. Ce faisant, la donation répondait aux besoins qu'avaient les institutions villageoises de trouver des sources de financement. Il fallait bien construire ou réparer les sanctuaires. Mais surtout, $\mathrm{du} \mathrm{XVI}^{\mathrm{e}} \mathrm{au}$ XVIII ${ }^{\mathrm{e}}$ siècle, les villages ont dû faire face aux dépenses colossales générées par l'obligation, venue de la cour, mais sans aucun subside, de construire des maisons communales (dinh).

De fait, la «donation intéressée », initiée au milieu du XvI ${ }^{\mathrm{e}}$ siècle, s'est imposée partout dans le courant $\mathrm{du}_{\mathrm{XVIII}}^{\mathrm{e}}$ siècle. À cette époque, elle était encore très marquée par la religion, ou au minimum par la rhétorique du discours religieux. Les inscriptions commencent toutes par de longs incipit bouddhistes qui glorifient la rencontre providentielle entre une personne souhaitant donner et une collectivité acceptant de recevoir; qui insistent sur la gratuité de la générosité des uns (投 桃, đầu đào) et de la gratitude des autres (報李, báo lý) ; qui décrivent la charité comme un bienfait gracieux, léger et naturel comme la pluie et la rosée (恩施雨露, ân thi vũ lọ); qui soulignent le devoir consistant à « secourir le peuple dans le besoin» (濟民之急, tế dân chi cấp). Mais, à partir de la seconde moitié du XIX ${ }^{\mathrm{e}}$ siècle, une fois la donation popularisée, passée à la portée d'à peu près tout le monde, le geste devient moins solennel. Le discours religieux diminue, jusqu'à disparaître parfois, et les textes entrent directement dans le vif du sujet: "Madame X offre telle somme d'argent et telles parcelles afin d'être honorée après sa mort du titre de Descendante de la divinité (Hậu) ». Mieux : on crée des Hậu non plus seulement pour la pagode et la maison communale mais pour n'importe quel lieu, par exemple un pâté de maisons (后甲, hậu giáp), une ruelle (后仵, hậu ngõ) ou encore, très surprenant, la collectivité toute entière (后公, hậu công). On voit même apparaître l'expression « acheter un titre de Descendant de la divinité ». La donation est devenue une procédure très simple, très ordinaire, quasiment assimilable à une taxe locale, et dès lors les stèles deviennent si nombreuses (et si peu précieuses) que certains villages, comme celui de Thuy-Khuê, les déposent pêlemêle dans un monument sommaire, semblable à un espace de stockage : le hậu điếm 店后 ou hậu đền 后祠. Avec le sanctuaire néo-confucéen de type văn chì, c'est un autre exemple de bâtiments nouveaux qui surgissent dans les villages sous la dynastie des Nguyễn.

C'est précisément la désacralisation du titre de Descendant divin, sa contamination par l'argent, l'économie et calcul, et par conséquent la suspicion généralisée qu'il a été acheté ou que son bénéficiaire a été taxé, qui entraînera, à la fin du XIX ${ }^{\mathrm{e}}$ siècle un mouvement très net de retour au ki ky. 寄忌, autrement dit à la forme simple de la donation : un geste purement individuel avec don de terre, sans argent ni titre honorifique, en vue d'assurer la commémoration funèbre du donateur. 
De fait, les textes sont riches en renseignements économiques et financiers. Ils précisent la nature, la quantité et le prix des choses. Quand on donne une parcelle, on en indique la localisation et la surface exacte. Quand on s'engage à organiser un banquet, on précise quels aliments il y aura sur la table et, souvent, la valeur de ces aliments ( « un porc valant $\mathrm{x}$ ligatures de sapèques »). Dès lors, stèle après stèle, année après année, village après village, nous pouvons reconstituer des listes entières de prix. Cette liste sert ensuite à évaluer le montant de la donation elle-même.

C'est ainsi qu'on peut déterminer, pour prendre l'exemple de l'inscription $\mathrm{n}^{\circ} 2760$, que telle donation de 30 ligatures de sapèques faite en 1770 dans tel village équivalait au prix de six buffles ou à dix mois de salaire d'un menuisier. Un sondage portant sur 144 inscriptions d'époque Nguyễn indique que la médiane des dons en numéraire s'établit à 100 ligatures (60000 sapèques). Mais le détail montre aussi une grande diversité ; en 1829, par exemple, il y a deux offrandes : l'une de 108800 sapèques, l'autre de 96000 ; en 1851, deux offrandes : 312000 et 10800 sapèques ; en 1903, cinq offrandes : 12 000, $36000,42000,120000$ et 156000 sapèques. Au bas de l'échelle, certains dons sont très modiques : Phạm Thị Di n'offre que 5 ligatures et Nguyễn Đình Huệ 10 ligatures (Lại Yên, 1809 et 1835, inscriptions n ${ }^{\text {os }} 1524-1525$ et 40379). En haut de l'échelle, on trouve de véritables fortunes : à Đại Lộ, en 1864, Đinh Thị Tư, originaire de Hanoi, fait une double offrande dont la valeur totale s'élève à 600 ligatures (修造碑記, no 26258); en 1889, à Vân Cốc, Nguyễn Phúc Bổng et sa femme donnent 700 ligatures pour la réparation de la maison communale (本亭碑記, $\mathrm{n}^{\circ}$ 31253). De 5 à 700 ligatures, la donation couvre ainsi tous les niveaux de richesse.

Ces renseignements chiffrés sont néanmoins abstraits, et trompeurs puisque la valeur de l'argent a changé - en l'occurrence, elle s'est dépréciée. C'est là où la conversion présente tout son intérêt. Elle permet de rapporter le niveau des donations à la valeur des produits, au moment où elles ont été faites. Prenons l'exemple de trois inscriptions du village de Vân Cốc : en 1814, un porc vaut 600 sapèques (後神碑記 /

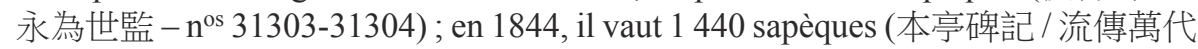
$-\mathrm{n}^{\mathrm{os}} 31243-31244$ ) ; en 1866, il vaut 1800 sapèques (天長地久 - $\mathrm{n}^{\mathrm{os}} 31317-31318$ ). Ainsi, dans ce village, le prix du porc a été multiplié par trois en cinquante ans. Dès lors, en terme de pouvoir d'achat, on peut considérer que la valeur de l'argent, elle, a été divisée par trois : 100 sapèques en 1866 valent 33 sapèques de 1814. On aboutit ainsi à des coefficients qui permettent de rétablir la valeur réelle des dons et de les comparer.

En règle générale, l'inflation a fait plus que doubler le prix du porc, une première fois de 1800 à 1850 , puis une seconde de 1850 à 1900 . Une valeur 100 en 1800 équivaut à une valeur 500 en 1900. Il faudrait donc multiplier les chiffres par cinq pour aboutir à la stabilité des valeurs. Or, comme au cours du XIX ${ }^{\mathrm{e}}$ siècle le don en espèces n'a pas été multiplié par cinq mais par deux, on en conclut que sa valeur réelle a baissé : en 1900, on donne certes deux fois plus d'argent facial qu'en 1800, mais cette somme doublée vaut 2,5 fois moins que la somme initiale. Le montant réel de la donation a chuté, les dons individuels ont baissé. Et s'ils ont baissé, c'est que la pratique de la donation s'est généralisée et que les autorités civiles et religieuses acceptaient désormais de petites sommes. 
Cette manière de compter présente encore l'avantage d'être très concrète. Dans le village de Vân Cốc, en 1844, le plus petit don est de 13000 sapèques et le plus important de 180000 : ce qui, sachant la valeur du porc cette année-là (1 440 sapèques), permet de dire que les donations représentaient une valeur allant de 10 à 140 porcs. On mesure mieux les choses, et on s'aperçoit en l'occurrence qu'à cette date il s'agissait encore de fortes sommes. Un élevage de 140 porcs représente une belle fortune. Quant aux petits dons, ils représentent quand même, au milieu du $\mathrm{XIX}^{\mathrm{e}}$ siècle, l'équivalent de deux, trois ou quatre cochons, ce qui n'est pas rien pour une famille paysanne ordinaire.

Évidemment, le calcul doit être mené de la même manière pour ce qui concerne les dons en terre. Le prix de celle-ci a varié et un sào de 1800 ne vaut pas le même prix qu'un sào de 1900. Cela dit, la transformation en équivalent réel est moins nécessaire parce que la terre possède une valeur agricole d'usage en elle-même, chaque famille ayant besoin d'une certaine surface pour produire le riz qui la fait vivre : cette surface et ce rendement sont à peu près constants sur un siècle de durée. La comparaison entre donations en est facilitée.

Les conférences se sont achevées par une synthèse financière des donations pieuses et l'ébauche d'un traitement quantitatif et informatique des données. 